\title{
电化学介导的芳香醛和脂肪醇氧化酯化反应
}

\author{
程诗砚 $a$ 欧楚鸿 ${ }^{a}$ 林洪敏 ${ }^{a}$ 贾均松 ${ }^{a}$ 唐海涛 $a$ \\ 潘英明 $a, c \quad$ 黄国保*,b 蒙秀金 $*, a$ \\ ( ${ }^{a}$ 广西师范大学化学与药学学院 省部共建药用资源化学与药物分子工程国家重点实验室 广西桂林 541004) \\ $(b$ 玉林师范学院化学与食品科学学院 广西农产资源化学与生物技术重点实验室 广西玉林 537000) \\ ( ${ }^{c}$ 吉首大学化学与化工学院 湖南吉首 416000)
}

\begin{abstract}
摘要 通过电氧化醛和醇合成了一系列酯类化合物. 该电合成反应在室温下即可进行, 无需惰性气体保护、外源氧化 剂、氮杂卡宾(NHC)催化剂和碱[如 DBU (1,5-二氮杂二环 [5.4.0]十一烯-5)等的参与. 反应采用简单易得的不分隔电解槽 和恒电流模式，具有反应条件温和、底物适用范围广、原料廉价易得和操作简便等优点，为酯类化合物的绿色合成提 供了一种新策略.
\end{abstract}

关键词 有机电合成; 脱氢偶联; 酯化反应

\section{Electrochemically Mediated Esterification of Aromatic Aldehydes with Aliphatic Alcohols via Anodic Oxidation}

\author{
Cheng, Shiyan ${ }^{a} \quad$ Ou, Chuhong ${ }^{a} \quad$ Lin, Hongmin ${ }^{a}$ \\ Jia, Junsong ${ }^{a}$ \\ Tang, Haitao ${ }^{a}$ \\ Pan, Yingming ${ }^{a, c} \quad$ Huang, Guobao ${ }^{*, b}$ Meng, Xiujin ${ }^{*, a}$ \\ ( ${ }^{a}$ State Key Laboratory for Chemistry and Molecular Engineering of Medicinal Resources, School of Chemistry and \\ Pharmaceutical Sciences, Guangxi Normal University, Guilin, Guangxi 541004) \\ $\left({ }^{b}\right.$ Key Laboratory of Agricultural Resources Chemistry and Biotechnology, College of Chemistry and Food Science, \\ Yulin Normal University, Yulin, Guangxi 537000) \\ ( ${ }^{c}$ College of Chemistry and Chemical Engineering, Jishou University, Jishou, Hunan 416000)
}

\begin{abstract}
A series of ester compounds were synthesized by direct oxidative coupling of aldehydes with alcohols. The electrosynthesis reaction can be carried out at room temperature without inert gas protection, external oxidants, N-heterocyclic carbene (NHC) catalyst and alkali (such as DBU (1,5-dizzabicyclo[5.4.0]undecen-5-ene)), etc. The oxidative esterification reaction processes in a simple and easily available undivided electrochemical cell and constant current mode. This protocol features mild reaction conditions, broad substrate scope, cheap and easily available starting materials, and simple operation, providing a new strategy for the green synthesis of ester compounds.
\end{abstract}

Keywords organic electrosynthesis; dehydrogenative coupling; esterification

酯基是有机合成中最重要和最丰富的官能团之一, 常用于生物活性化合物的合成砌块和保护基团 ${ }^{[1]}$, 普遍 存在于天然产物和药物中. 例如, 芳香酯类化合物广泛 应用于香料、香醋、溶剂、化妆品、中间体等 ${ }^{[2-3]}$. 图 1 列举了一些常见的市售芳香酯香料: 杏仁味的苯甲酸甲
酯、草莓味的肉桂酸甲酯、玫瑰味的 4-硝基苯甲酸香叶 酯、香蕉味的苯甲酸丁酯和著名的、带有 Iodex 气味的 2-水杨酸甲酯油 ${ }^{[4]}$. 此外，内酯类化合物还应用于药物 治疗，如第一批应用于 HMG-Co A 还原酶抑制剂的 “美 伐他汀(Mevastatin)” (图 1) ${ }^{[5]}$.

\footnotetext{
* Corresponding authors. E-mail: mengxiujin@163.com; 1zjx0915@163.com

Received October 14, 2021; revised December 8, 2021; published online December 15, 2021.

Project supported by the Guangxi Natural Science Foundation (No. 2021GXNSFBA196041), the Guangxi Key Laboratory of Agricultural Resources Chemistry and Biotechnology (No. 2020KF04) and the Opening Project of Hunan Engineering Laboratory for Analysis and Drugs Development of Ethnomedicine in Wuling Mountains (No. hgxy2101).

广西自然科学基金(No. 2021GXNSFBA196041)、广西农产资源化学与生物技术重点实验室开放课题基金(No. 2020KF04)、武陵山地区民族药解析与 创制湖南省工程实验室开放课题(No. hgxy2101)资助项目.
} 
醛和醇通过氧化酯化合成酯已有大量文献报道，常 见的方法是利用贵金属(如 $\mathrm{Au}, \mathrm{Pd}, \mathrm{Rh}$ 等)作为催化剂 ${ }^{[6]}$, 然而, 这种策略往往需要高温、高压(氧气氛围)环境, 反 应条件较为苛刻且耗时长, 存在一定的安全隐患. 氮杂 卡宾(NHC)催化策略也被用于醛和醇的氧化酯化反应 ${ }^{[7]}$, 然而这种策略通常还需要过量的氧化剂, 容易发生副反 应. 此外, 无金属催化策略也被用于醛和醇的氧化酯化 反应，但是这种策略一般都需要过量强酸或毒性极强的 添加剂 ${ }^{[8]}$, 严重限制了底物适用范围. 因此, 开发一种 反应条件温和、绿色简洁的有机电化学合成策略来实现 醛和醇的氧化酯化反应仍然具有挑战性.

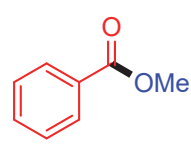

Marzipan smell

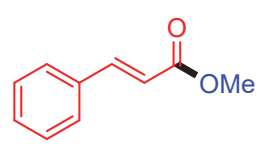

Strawberry flavor

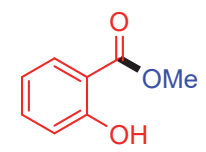

Oil of Winter Green<smiles>CCCCOC(=O)c1ccccc1</smiles>

Banana Flavour

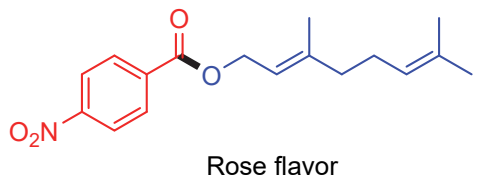

Rose flavor<smiles>CC[C@H](C)C(=O)O[C@H]1CCC=C2C=C[C@H](C)[C@H](CC[C@@H]3C[C@@H](O)CC(=O)O3)[C@H]21</smiles>

Mevastatin

图 1 部分市售芳香酯香料以及 HMG-Co A 还原酶抑制剂 “Mevastain”

Figure 1 Some common commercially available aromatic esters spices and the HMG-Co A reductase inhibitor "Mevastain"

近年来, 有机电化学合成技术引起化学工作者广泛 的关注 ${ }^{[9]}$, 因为该技术利用电子作为氧化剂或还原剂, 无需外源氧化剂或还原剂, 具有原子经济、绿色环保等 显著优势. Boydston 课题组 ${ }^{[10]}$ 将有机电合成手段与 NHC 催化相结合, 在氮气保护和恒电位阳极氧化条件下, 实 现了醛和醇的氧化酯化反应 (Scheme 1a). 类似地, Brown 课题组 ${ }^{[11]}$ 在流动电解池中通过恒电流阳极氧化 也实现了醛和醇的氧化酯化反应(该反应电流高达 850 $\mathrm{mA}$, Scheme 1a). 原位生成 NHC 催化剂进而得到 Breslow 中间体是这两种策略成功的关键步骤, 然而反 应必须在有机碱 DBU 和惰性气体保护或高电流密度协 同作用下才可以实现, 反应条件还不够温和. 根据我们 在有机电化学交叉脱氢偶联的工作经验 ${ }^{[12]}$, 设想是否 可以在恒电流模式下不需要经过 NHC 催化这种机理,
也能一步实现醛和醇的氧化酯化反应(Scheme 1b)?

(a) Previous work:

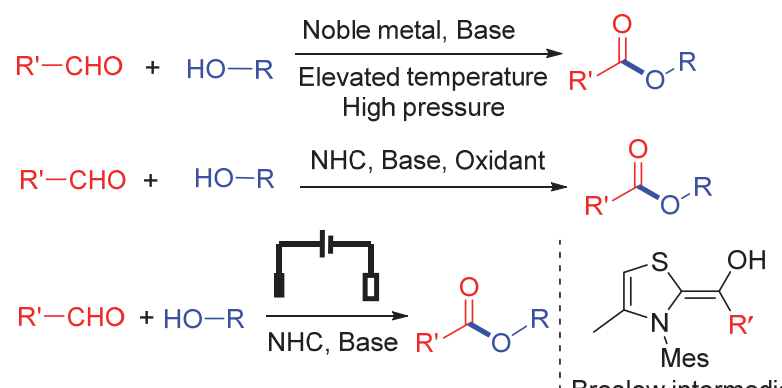

(b) This work:

Breslow intermediate

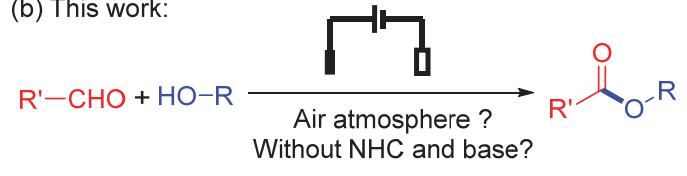

图式 1 醛和醇的氧化酯化反应

Scheme 1 Oxidative esterification of aldehydes and alcohols

\section{1 结果与讨论}

\section{1 反应条件的优化}

首先, 以茴香醛 $(1 a)$ 和甲醇 $(2 a)$ 为底物, 对反应的 电流、电解质、溶剂以及电极进行了篮选(表 1). 当网状 玻璃态碳电极 (RVC) 作为阳极, 铂片作为阴极, 电流为 5 $\mathrm{mA}$ 时，目标产物 3a 产率为 65\%(表 1, Entry 1). 值得注 意的是, 当电流为 $10 \mathrm{~mA}$ 时, 目标产物 $\mathbf{3 a}$ 产率提高为 $92 \%$ (表 1, Entry 2). 当电流由 $10 \mathrm{~mA}$ 变为 $15 \mathrm{~mA}$ 时, 目 标产物 3a 的产率没有变化(表 1, Entry 3). 当以碘化铵 $\left(\mathrm{NH}_{4} \mathrm{I}\right)$ 为电解质, 目标产物 $3 \mathbf{a}$ 的产率降至 $35 \%$ (表 1 , Entry 4). 当选择四丁基碘化铵 $\left(n-\mathrm{Bu}_{4} \mathrm{NI}\right)$ 作为电解质时, 目标产物 3a 的产率仅为 30\%(表 1, Entry 5). 当以四丁基 高氯酸铵 $\left(n-\mathrm{Bu}_{4} \mathrm{NClO}_{4}\right)$ 为电解质时, 没有观察到目标产 物 3a 的生成(表 1, Entry 6). 随后篮选了反应的溶剂, 当 以二甲基亚砜(DMSO)为溶剂时，目标产物 $3 \mathbf{a}$ 产率为 $58 \%$ (表 1, Entry 7). 当以 $N, N$-二甲基甲酰胺(DMF)为反 应溶剂时，仅观察到痕量 3a 的生成(表 1, Entry 8). 当以 二氯甲烷(DCM) 为溶剂时，仅得到产率 $20 \%$ 的 $3 \mathrm{a}$ (表 1 , Entry 9). 最后, 还对反应电极进行篮选, 当以铂片作为 阳极, RVC 作为阴极时, 目标产物 $\mathbf{3 a}$ 的产率仅为 25\%(表 1, Entry 10). 当选用其他电极如铁电极、镍电 极、碳毡作为阴极时, 目标产物 $3 \mathbf{a}$ 的产率也不理想(表 1, Entries $11 \sim 13)$. 此外，当以 RVC 同时作为阳极和阴 极时，目标产物 3a 的产率为 45\%(表 1, Entry 14). 当以 石墨棒作为阳极, 铂片作为阴极时, 目标产物 $\mathbf{3 a}$ 产率为 $85 \%$ (表 1, Entry 15). 当以铂片同时作为阳极和阴极时, 没有观察到目标产物 3a 产生(表 1, Entry 16). 综上所述, 该反应最佳的条件是：以乙腈为溶剂，恒定电流为 10.0 
表 1 合成酯类化合物的反应条件优化 ${ }^{a}$

Table 1 Reaction optimization for the synthesis of esters<smiles>COC(=O)c1ccc(OCC(=O)OCCOc2ccc(C=O)cc2)cc1</smiles>

\begin{tabular}{|c|c|c|c|c|c|}
\hline Entry & Electrolyte & Solvent & $\begin{array}{c}\text { Current/ } \\
\mathrm{mA}\end{array}$ & Electrode & $\begin{array}{c}\text { Yield }^{b} / \\
\% \\
\end{array}$ \\
\hline 1 & $\mathrm{LiClO}_{4}$ & $\mathrm{CH}_{3} \mathrm{CN}$ & 5 & $\operatorname{RVC}(+) \| \operatorname{Pt}(-)$ & 65 \\
\hline 2 & $\mathrm{LiClO}_{4}$ & $\mathrm{CH}_{3} \mathrm{CN}$ & 10 & $\operatorname{RVC}(+) \| \operatorname{Pt}(-)$ & 92 \\
\hline 3 & $\mathrm{LiClO}_{4}$ & $\mathrm{CH}_{3} \mathrm{CN}$ & 15 & $\operatorname{RVC}(+) \| \operatorname{Pt}(-)$ & 92 \\
\hline 4 & $\mathrm{NH}_{4} \mathrm{I}$ & $\mathrm{CH}_{3} \mathrm{CN}$ & 10 & $\operatorname{RVC}(+) \| \operatorname{Pt}(-)$ & 35 \\
\hline 5 & TBAI & $\mathrm{CH}_{3} \mathrm{CN}$ & 10 & $\operatorname{RVC}(+) \| \operatorname{Pt}(-)$ & 30 \\
\hline 6 & TBAP & $\mathrm{CH}_{3} \mathrm{CN}$ & 10 & $\operatorname{RVC}(+) \| \operatorname{Pt}(-)$ & NR \\
\hline 7 & $\mathrm{LiClO}_{4}$ & DMSO & 10 & $\mathrm{RVC}(+) \| \operatorname{Pt}(-)$ & 58 \\
\hline 8 & $\mathrm{LiClO}_{4}$ & DMF & 10 & $\mathrm{RVC}(+) \| \operatorname{Pt}(-)$ & Trace \\
\hline 9 & $\mathrm{LiClO}_{4}$ & DCE & 10 & $\mathrm{RVC}(+) \| \operatorname{Pt}(-)$ & 20 \\
\hline 10 & $\mathrm{LiClO}_{4}$ & $\mathrm{CH}_{3} \mathrm{CN}$ & 10 & $\operatorname{Pt}(-) \| \operatorname{RVC}(+)$ & 25 \\
\hline 11 & $\mathrm{LiClO}_{4}$ & $\mathrm{CH}_{3} \mathrm{CN}$ & 10 & $\mathrm{RVC}(+) \| \mathrm{Fe}(-)$ & 30 \\
\hline 12 & $\mathrm{LiClO}_{4}$ & $\mathrm{CH}_{3} \mathrm{CN}$ & 10 & $\mathrm{RVC}(+) \| \mathrm{Ni}(-)$ & 38 \\
\hline 13 & $\mathrm{LiClO}_{4}$ & $\mathrm{CH}_{3} \mathrm{CN}$ & 10 & $\operatorname{RVC}(+) \| \mathrm{C}$ felt( $(-)$ & 40 \\
\hline 14 & $\mathrm{LiClO}_{4}$ & $\mathrm{CH}_{3} \mathrm{CN}$ & 10 & $\operatorname{RVC}(+) \| \operatorname{RVC}(-)$ & 45 \\
\hline 15 & $\mathrm{LiClO}_{4}$ & $\mathrm{CH}_{3} \mathrm{CN}$ & 10 & $\mathrm{C}(+) \| \operatorname{Pt}(-)$ & 85 \\
\hline 16 & $\mathrm{LiClO}_{4}$ & $\mathrm{CH}_{3} \mathrm{CN}$ & 10 & $\operatorname{Pt}(+) \| \operatorname{Pt}(-)$ & NR \\
\hline
\end{tabular}

${ }^{a}$ Reaction conditions: undivided cell, 1a $(0.5 \mathrm{mmol}), 2 \mathrm{a}(1.5 \mathrm{mmol}, 3.0$ equiv.), $\mathrm{LiClO}_{4}\left(0.25 \mathrm{mmol}, 0.5\right.$ equiv.), $\mathrm{CH}_{3} \mathrm{CN}(5.0 \mathrm{~mL})$, room temperature;

${ }^{b}$ Isolated yield. $\mathrm{NR}=$ no reaction.
$\mathrm{mA}, 0.5$ equiv.的 $\mathrm{LiClO}_{4}$ 作为电解质, RVC 作为阳极, 铂 片为阴极. 这可能是因为 RVC 电极有高的表面积, 有利 于电子转移，而铂片作为阴极有利于氢析出，因此反应 效果最好.

\section{2 醛与醇的反应}

得到最佳反应条件后, 研究了该反应的底物普适范 围(表 2). 首先，对一系列直链型的烷基醇进行了考察. 当选用乙醇作为底物时, 该反应在 $2 \mathrm{~h}$ 左右即可完成, 且目标产物产率达 90\%(表 2, 3b). 当继续增加碳链的长 度，分别以正丙醇、正丁醇和正戊醇为底物时，也能够 以较高的产率获得目标产物，产率分别为 $85 \% 、 80 \%$ 、 $72 \%$ (表 2, 3c、3e、3g)，然而，随着烷基醇碳链的增长，反 应所需时间逐渐增加. 当选择带有支链结构的醇为底物 时，如异丙醇和叔丁醇，目标产物会随着碳个数的增加 而急剧下降，产率分别为 $70 \% 、 35 \%$ (表 $2,3 \mathbf{3 d} 、 3 \mathrm{f}$ ). 当以 乙二醇甲醚为底物时，目标产物产率令人满意，达到 $82 \%$ (表 2, 3h). 当以炔丙醇为底物时, 产物产率为 $45 \%$ (表 2, 3i). 当以三氟乙醇为底物时, 产物产率为 35\%(表 $2,3 \mathbf{j}$ ). 随后，对环烷烃醇进行考察. 当以环己醇为底物 时，以 $32 \%$ 的产率获得目标产物 $3 \mathbf{k}$ (表 2). 当以薄荷醇 为底物时, 以 $70 \%$ 的产率获得目标产物(表 2,31 ). 当以

表 2 反应底物适用范围 ${ }^{a}$

Table 2 Reaction scope for the substrate<smiles>[R]OC(=O)c1ccc([R])cc1</smiles><smiles>CCCCOC(=O)c1ccc(OC)cc1</smiles><smiles>COc1ccc(C(=O)OC(C)(C)C)cc1</smiles>

3f, $35 \%$<smiles>CCCCCOC(=O)c1ccc(OC)cc1</smiles>

3g, $72 \%$<smiles>COCCOC(=O)c1ccc(OC)cc1</smiles>

3h, $82 \%$<smiles>C#CCOC(=O)c1ccc(OC)cc1</smiles>

$3 i, 45 \%$<smiles>COc1ccc(C(=O)OCC(F)(F)F)cc1</smiles>

3j, $35 \%$<smiles>COc1ccc(C(=O)OC2CCCCC2)cc1</smiles>

3k, $32 \%$<smiles>CC(C)[C@H]1CCCC[C@H]1OC(=O)c1ccc(O)cc1</smiles>

3I, $70 \%$<smiles></smiles>

$3 \mathrm{~m}, 67 \%$<smiles>CCOC(=O)c1ccc(C(C)(C)C)cc1</smiles>

3o, $85 \%$<smiles>CCOC(=O)c1ccc(Cl)cc1</smiles>

$3 p, 53 \%$<smiles>CCOC(=O)c1ccc(Br)cc1</smiles>

3q, $67 \%$<smiles>CCOC(=O)c1ccc(C=O)cc1</smiles>

3 r, $50 \%$<smiles>COc1ccc(C(=O)OC2C[C@H]3CC[C@H](C2)C3C)cc1</smiles>

3n, $42 \%$<smiles>C#Cc1ccc(C(=O)OCC)cc1</smiles>

3s, $58 \%$

${ }^{a}$ Reaction conditions: undivided cell with RVC anode and Pt plate cathode, constant current $=10 \mathrm{~mA}, 1$ (0.5 mmol, 1.2 equiv.), 2 (1.5 mmol, 3.0 equiv.), LiClO ( 0.25 mmol, 0.5 equiv.), and $\mathrm{CH}_{3} \mathrm{CN}(5 \mathrm{~mL})$, room temperature. ${ }^{b}$ Isolated yield. 
金刚烷甲醇为底物时, 也能以 $67 \%$ 的产率获得目标产物 (表 2, 3m). 当以冰片为底物时, 目标产物产率较低, 仅 为 $42 \%$, 这可能是因为冰片较大的空间位阻导致(表 2 , 3n).

紧接着, 以乙醇为醇类底物, 考察其它醛类化合物 对该策略的适用性. 首先对带有供电子基团的叔丁基苯 甲醛进行研究, 发现可以以 $85 \%$ 的产率获得目标产物 (表 2,30). 随后, 对带有吸电子基团的苯甲醛进行研究, 当分别以对氯、对溴苯甲醛为底物时, 产率中等, 分别 为 $53 \% 、 67 \%$ (表 $2,3 \mathbf{3 p} \sim 3 \mathbf{q}$ ). 当选择对苯二甲醛作为底 物时, 也以中等产率获得预期目标产物(表 2, 3r). 此外, 带有不饱和参键的苯甲醛也适用于该策略, 以 $58 \%$ 的中 等产率获得目标产物(表 2, 3s). 综上可知, 该策略具有 较广泛的底物适用范围, 而且反应条件温和, 无需惰性 气体保护, 也不需要添加任何 NHC 催化剂和碱, 室温 下即可顺利进行.

\section{3 控制实验}

为了深入了解这个反应机理, 进行了一系列控制实 验(Scheme 2). 当茴香醛(1a)和甲醇 (2a) 在不通电、其它 条件参照标准条件时, 该反应不发生, 未观察到任何产 物 3a 生成, 说明电流在该反应中起关键作用(Scheme 2, a). 为了验证是否是甲氧根负离子进攻茴香醛的羰基 碳、再经过后续转化从而获得目标产物 3a? 选择纯乙腈 或二甲基亚砜作为溶剂, 甲醇钠(4)作为甲氧根负离子 源, 在标准条件下对茴香醛(1a)和甲醇钠(4)进行电解,

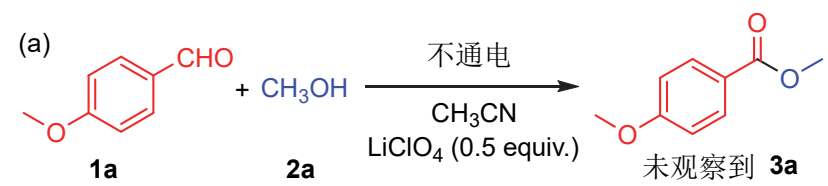

(b)
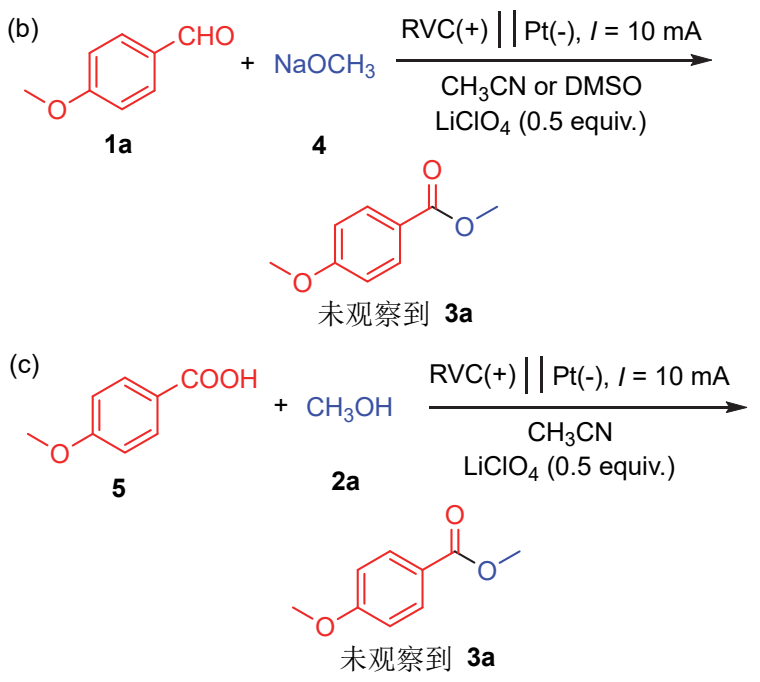

图式 2 控制实验

Scheme 2 Control experiments
也未观察到任何产物 3a 生成(Scheme 2, b). 为了验证茴 香醛(1a) 是否是在阳极先氧化为酸后再与醇发生酯化反 应? 选择对甲氧基苯甲酸(5)与甲醇 (2a)作为底物在标准 条件下进行电解，但是并没有检测到目标产物 $\mathbf{3 a}$ (Scheme 2, c), 这个结果表明茴香醛并没有在阳极氧化 为酸.

\section{4 反应机制}

基于以上控制实验结果和文献报道 ${ }^{[13]}$, 我们提出 该反应在电化学条件下可能的机理(图 2), 首先, 醛 $\mathbf{1}$ 在 阳极通过电子转移氧化生成自由基阳离子 $\mathbf{A}$, 随后中间 体 $\mathbf{A}$ 失去一个氢原子得到稳定的酰基阳离子 $\mathbf{B}$ ，中间体 $\mathbf{B}$ 再与醇 2 发生亲核加成反应得到中间体 $\mathbf{C}$ ，中间体 $\mathbf{C}$ 再经过一个脱质子过程得到目标产物 3 . 同时, 氢正离 子在阴极得到电子变为氢气, 完成整个反应的循环.

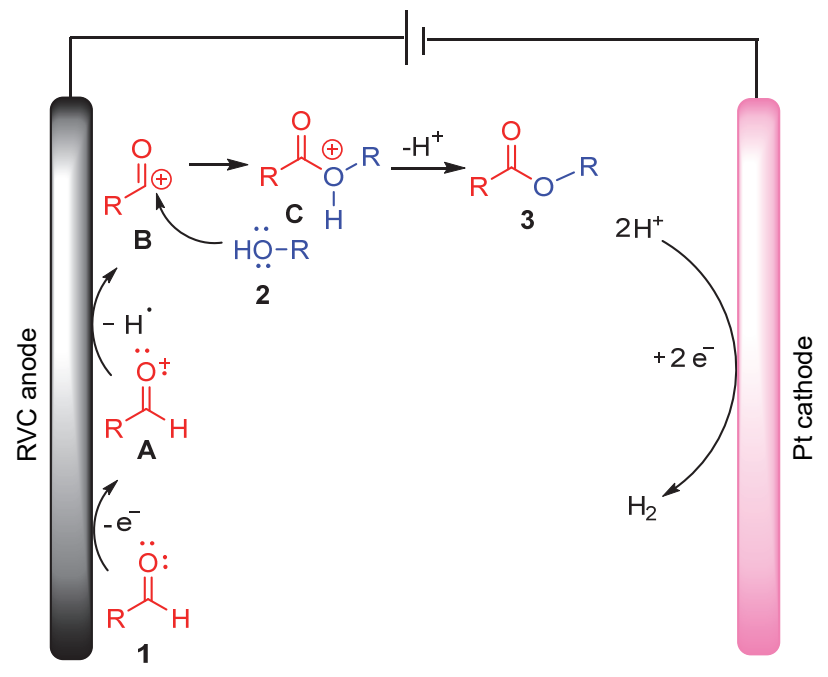

图 2 可能的反应机理

Figure 2 Possible reaction mechanism

\section{2 结论}

本文报道了一种简便的有机电化学合成方法合成 酯类化合物，以醛和醇为原料，反应采用恒电流模式， 反应在室温、大气环境下即可进行, 无需惰性气体保护、 金属催化剂, 也不需要添加 NHC 催化剂和碱, 具有反 应条件温和，操作简便，原料廉价易得，底物普适性广 等优点, 为醛和醇氧化酯化合成酯类化合物提供了一种 绿色简便的合成策略.

\section{3 实验部分}

\section{1 仪器与试剂}

柱层析过程中使用的硅胶来自青岛海洋化工集团 公司 (300 400 目, 化学纯), 薄层色谱(TLC)所用薄层 层析板为该公司生产的 GF-254 板. 核磁共振光谱 
(NMR)在 Bruker Avance III HD (400 MHz)或 Bruker Avance NEO (600 MHz)上进行, 以氛代 $\mathrm{CDCl}_{3}$ 和氛代 DMSO- $d_{6}$ 为溶剂, 以四甲基硅烷(TMS)为内标. 通过 Agilent 6545 Q-TOF LC/MS 联用高分辨质谱仪(ESI)测 定 HRMS 谱. 实验所用各类试剂均为分析纯, 液体试剂 如有必要都会进行干燥处理, 所有实验药品在泰坦集团 化学试剂有限公司、北京伊诺凯科技有限公司等购买, 均为分析纯.

\section{2 实验方法}

将醛 $1(0.5 \mathrm{mmol}) 、$ 醇 $\mathbf{2}(3 \mathrm{~mL}) 、 \mathrm{LiClO}_{4}(0.25 \mathrm{mmol}$, 0.5 equiv.)、乙腈 $(3 \mathrm{~mL})$ 依次加入 $10 \mathrm{~mL}$ 三颈瓶中, $\mathrm{RVC}$ $(100 \mathrm{PPI})$ 作为阳极电极材料, 铂片 $(1 \mathrm{~cm} \times 1 \mathrm{~cm} \times 0.1$ $\mathrm{cm}$ )作为阴极电极材料, 恒电流设置为 $10 \mathrm{~mA}$, 在室温 $\left(25{ }^{\circ} \mathrm{C}\right.$ 左右)下进行电解, 通过薄层色谱法监测整个反 应过程直至底物完全消耗. 反应结束后, 用乙酸乙酯和 饱和食盐水萃取反应液, 用无水 $\mathrm{Na}_{2} \mathrm{SO}_{4}$ 干燥并在减压 蒸馏条件减压下除去溶剂. 用硅胶柱色谱法纯化, 用乙 酸乙酯/石油醚洗脱剂洗脱得到目标产物 3.

\section{3 目标化合物结构表征}

4-甲氧基苯甲酸甲酯 $(3 a)^{[14]}$ : 无色液体, 产率 $92 \%$. ${ }^{1} \mathrm{H}$ NMR $\left(400 \mathrm{MHz}, \mathrm{CDCl}_{3}\right) \delta: 8.02 \sim 7.96(\mathrm{~m}, 2 \mathrm{H})$, $6.94 \sim 6.89$ (m, 2H), 3.88 (s, 3H), 3.85 (s, 3H); ${ }^{13} \mathrm{C}$ NMR $\left(100 \mathrm{MHz}, \mathrm{CDCl}_{3}\right) \delta: 165.8,162.3,130.6,121.6,112.6$, 54.4, 50.8; HRMS (ESI) calcd for $\mathrm{C}_{9} \mathrm{H}_{11} \mathrm{O}_{3}[\mathrm{M}+\mathrm{H}]^{+}$ 167.0703, found 167.0705.

4-甲氧基苯甲酸乙酯 $(\mathbf{3 b})^{[15]}$ : 无色液体，产率 $90 \%$. ${ }^{1} \mathrm{H}$ NMR $\left(400 \mathrm{MHz}, \mathrm{CDCl}_{3}\right) \delta: 8.04 \sim 7.93(\mathrm{~m}, 2 \mathrm{H})$, $6.94 \sim 6.86(\mathrm{~m}, 2 \mathrm{H}), 4.34(\mathrm{q}, J=7.06 \mathrm{~Hz}, 2 \mathrm{H}), 3.85$ (s, $3 \mathrm{H}), 1.37(\mathrm{t}, J=7.07 \mathrm{~Hz}, 3 \mathrm{H}) ;{ }^{13} \mathrm{C}$ NMR $(100 \mathrm{MHz}$, $\left.\mathrm{CDCl}_{3}\right) \delta: 166.4,163.2,131.5,122.9,113.5,60.6,55.4$, 14.3; HRMS (ESI) calcd for $\mathrm{C}_{10} \mathrm{H}_{13} \mathrm{O}_{3}[\mathrm{M}+\mathrm{H}]^{+}$ 181.0859 , found 181.0862 .

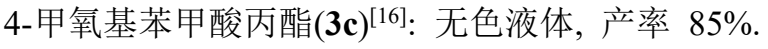
${ }^{1} \mathrm{H}$ NMR $\left(400 \mathrm{MHz}, \mathrm{CDCl}_{3}\right) \delta: 8.07 \sim 7.93(\mathrm{~m}, 2 \mathrm{H})$, $6.95 \sim 6.85(\mathrm{~m}, 2 \mathrm{H}), 4.24(\mathrm{t}, J=6.64 \mathrm{~Hz}, 2 \mathrm{H}), 3.85(\mathrm{~s}, 3 \mathrm{H})$, $1.82 \sim 1.72(\mathrm{~m}, 2 \mathrm{H}), 1.01(\mathrm{t}, J=7.42 \mathrm{~Hz}, 3 \mathrm{H}) ;{ }^{13} \mathrm{C}$ NMR $\left(100 \mathrm{MHz}, \mathrm{CDCl}_{3}\right) \delta: 166.4,163.2,131.5,122.9,113.5$, 66.2, 55.4, 22.1, 10.5; HRMS (ESI) calcd for $\mathrm{C}_{11} \mathrm{H}_{15} \mathrm{O}_{3}$ $[\mathrm{M}+\mathrm{H}]^{+}$195.1016, found 195.1019.

4- 甲氧基苯甲酸异丙酯 $(3 d)^{[17]}$ : 无色液体, 产率 $70 \% .{ }^{1} \mathrm{H}$ NMR (400 MHz, $\left.\mathrm{CDCl}_{3}\right) \delta: 8.02 \sim 7.97(\mathrm{~m}, 2 \mathrm{H})$, $6.93 \sim 6.89(\mathrm{~m}, 2 \mathrm{H}), 5.26 \sim 5.18(\mathrm{~m}, 1 \mathrm{H}), 3.86(\mathrm{~s}, 3 \mathrm{H})$, $1.36(\mathrm{~s}, 3 \mathrm{H}), 1.34(\mathrm{~s}, 3 \mathrm{H}) ;{ }^{13} \mathrm{C} \mathrm{NMR}\left(100 \mathrm{MHz}, \mathrm{CDCl}_{3}\right) \delta$ : 165.9, 163.2, 131.5, 123.4, 113.5, 67.9, 55.4, 22.0; HRMS (ESI) calcd for $\mathrm{C}_{11} \mathrm{H}_{14} \mathrm{O}_{3} \mathrm{Na}[\mathrm{M}+\mathrm{Na}]^{+}$217.0835, found
217.0831.

4-甲氧基苯甲酸丁酯(3e) ${ }^{[15]}$ : 无色液体，产率 $85 \%$. ${ }^{1} \mathrm{H}$ NMR $\left(400 \mathrm{MHz}\right.$, DMSO- $\left.d_{6}\right) \delta: 7.93 \sim 7.88(\mathrm{~m}, 2 \mathrm{H})$, $7.06 \sim 7.02(\mathrm{~m}, 2 \mathrm{H}), 4.23(\mathrm{t}, J=6.55 \mathrm{~Hz}, 2 \mathrm{H}), 3.83(\mathrm{~s}, 3 \mathrm{H})$, $1.70 \sim 1.63(\mathrm{~m}, 2 \mathrm{H}), 1.46 \sim 1.37(\mathrm{~m}, 2 \mathrm{H}), 0.92(\mathrm{t}, J=7.39$ $\mathrm{Hz}, 3 \mathrm{H}) ;{ }^{13} \mathrm{C}$ NMR $\left(100 \mathrm{MHz}\right.$, DMSO- $\left.d_{6}\right) \delta: 165.4,163.1$, $131.2,122.1,114.0,64.0,55.5,30.3,18.8,13.6$; HRMS (ESI) calcd for $\mathrm{C}_{12} \mathrm{H}_{17} \mathrm{O}_{3}[\mathrm{M}+\mathrm{H}]^{+}$209.1172, found 209.1172

4- 甲氧基苯甲酸叔丁酯 $(\mathbf{3 f})^{[18]}$ : 无色液体，产率 $35 \%$. ${ }^{1} \mathrm{H}$ NMR (600 MHz, DMSO- $\left.d_{6}\right) \delta: 7.85 \sim 7.83(\mathrm{~m}$, $2 \mathrm{H}), 7.02 \sim 7.00(\mathrm{~m}, 2 \mathrm{H}), 3.81(\mathrm{~s}, 3 \mathrm{H}), 1.52(\mathrm{~s}, 9 \mathrm{H}) ;{ }^{13} \mathrm{C}$ NMR $\left(150 \mathrm{MHz}\right.$, DMSO- $\left.d_{6}\right) \delta: 164.7,162.8,131.1,123.6$, 113.8, 80.1, 55.5, 27.9; HRMS (ESI) calcd for $\mathrm{C}_{12} \mathrm{H}_{17} \mathrm{O}_{3}$ $[\mathrm{M}+\mathrm{H}]^{+}$209.1178, found 209.1172.

4-甲氧基苯甲酸戊酯 $(3 g)^{[19]}$ : 无色液体，产率 $72 \%$. ${ }^{1} \mathrm{H}$ NMR $\left(400 \mathrm{MHz}, \mathrm{CDCl}_{3}\right) \delta: 8.03 \sim 7.96(\mathrm{~m}, 2 \mathrm{H})$, $6.94 \sim 6.89(\mathrm{~m}, 2 \mathrm{H}), 4.28(\mathrm{t}, J=6.52 \mathrm{~Hz}, 2 \mathrm{H}), 3.86(\mathrm{~s}, 3 \mathrm{H})$, $1.78 \sim 1.72(\mathrm{~m}, 2 \mathrm{H}), 1.44 \sim 1.37(\mathrm{~m}, 4 \mathrm{H}), 0.92(\mathrm{t}, J=6.55$ $\mathrm{Hz}, 3 \mathrm{H}) ;{ }^{13} \mathrm{C} \mathrm{NMR}\left(100 \mathrm{MHz}, \mathrm{CDCl}_{3}\right) \delta: 166.5,163.2$, $131.5,122.9,113.5,64.8,55.4,28.5,28.2,22.4,14.0$; HRMS (ESI) calcd for $\mathrm{C}_{13} \mathrm{H}_{19} \mathrm{O}_{3}[\mathrm{M}+\mathrm{H}]^{+}$223.1329, found 223.1331 .

4-甲氧基苯甲酸 2-甲氧基乙酯 $(\mathbf{3 h})^{[20]}$ : 无色液体, 产率 82\%. ${ }^{1} \mathrm{H}$ NMR $\left(400 \mathrm{MHz}, \mathrm{CDCl}_{3}\right) \delta: 8.05 \sim 7.99(\mathrm{~m}$, $2 \mathrm{H}), 6.93 \sim 6.88(\mathrm{~m}, 2 \mathrm{H}), 4.44(\mathrm{t}, J=4.77 \mathrm{~Hz}, 2 \mathrm{H}), 3.85(\mathrm{~s}$, $3 \mathrm{H}), 3.71(\mathrm{t}, J=4.65 \mathrm{~Hz}, 2 \mathrm{H}), 3.42(\mathrm{~s}, 3 \mathrm{H}) ;{ }^{13} \mathrm{C}$ NMR $(100$ $\left.\mathrm{MHz}, \mathrm{CDCl}_{3}\right) \delta: 166.3,163.4,131.7,122.4,113.5,70.6$, 63.7, 59.0, 55.4; HRMS (ESI) calcd for $\mathrm{C}_{11} \mathrm{H}_{15} \mathrm{O}_{4}[\mathrm{M}+\mathrm{H}]^{+}$ 233.0784, found 233.0781.

4-甲氧基苯甲酸丙-2-炔-1-基 $(3 \mathbf{i})^{[21]}$ : 无色液体，产 率 45\%. ${ }^{1} \mathrm{H}$ NMR (400 MHz, DMSO- $d_{6}$ ) $\delta: 7.94 \sim 7.91$ (m, $2 \mathrm{H}), 7.08 \sim 7.01(\mathrm{~m}, 2 \mathrm{H}), 4.91(\mathrm{~d}, J=2.43 \mathrm{~Hz}, 2 \mathrm{H}), 3.84$ (s, 3H), $3.59(\mathrm{t}, J=2.39 \mathrm{~Hz}, 1 \mathrm{H}) ;{ }^{13} \mathrm{C}$ NMR $(100 \mathrm{MHz}$, DMSO- $\left.d_{6}\right) \delta$ : 164.6, 163.4, 131.4, 121.1, 114.2, 78.6, 77.8, 55.6, 52.1; HRMS (ESI) calcd for $\mathrm{C}_{11} \mathrm{H}_{10} \mathrm{O}_{3} \mathrm{Na}[\mathrm{M}+\mathrm{Na}]^{+}$ 213.0522, found 213.0519.

4-甲氧基苯甲酸 $2,2,2$-三氟乙酯 $(\mathbf{3 j})^{[22]}$ : 无色液体, 产率 35\%. ${ }^{1} \mathrm{H}$ NMR (600 MHz, DMSO- $d_{6}$ ) $\delta: ~ 7.97 \sim 7.94$ $(\mathrm{m}, 2 \mathrm{H}), 7.11 \sim 7.09(\mathrm{~m}, 2 \mathrm{H}), 4.96(\mathrm{q}, J=9.08 \mathrm{~Hz}, 2 \mathrm{H})$, $3.85(\mathrm{~s}, 3 \mathrm{H}) ;{ }^{13} \mathrm{C}$ NMR $\left(150 \mathrm{MHz}, \mathrm{DMSO}-d_{6}\right) \delta: 163.9$, $163.9,131.8,125.6$ (q, $J=277.29 \mathrm{~Hz}$ ), 120.1, 114.4, 60.1 (q, $J=35.09 \mathrm{~Hz}$ ), 55.7; HRMS (ESI) calcd for $\mathrm{C}_{10} \mathrm{H}_{10} \mathrm{~F}_{3} \mathrm{O}_{3}$ $[\mathrm{M}+\mathrm{H}]^{+}$235.0577, found 235.0570.

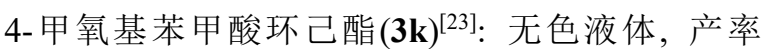


$32 \% .{ }^{1} \mathrm{H}$ NMR $\left(600 \mathrm{MHz}, \mathrm{DMSO}-d_{6}\right) \delta: 7.92 \sim 7.89(\mathrm{~m}$, $2 \mathrm{H}), 7.0 \sim 7.02(\mathrm{~m}, 2 \mathrm{H}), 3.82(\mathrm{~s}, 3 \mathrm{H}), 1.87 \sim 1.84(\mathrm{~m}, 2 \mathrm{H})$, $1.73 \sim 1.69(\mathrm{~m}, 2 \mathrm{H}), 1.55 \sim 1.48(\mathrm{~m}, 4 \mathrm{H}), 1.42 \sim 1.37(\mathrm{~m}$, $2 \mathrm{H}) ;{ }^{13} \mathrm{C}$ NMR (150 MHz, DMSO- $\left.d_{6}\right) \delta: 164.8,163.1$, 131.2, 122.5, 114.0, 72.0, 55.5, 31.1, 25.0, 23.1; HRMS (ESI) calcd for $\mathrm{C}_{14} \mathrm{H}_{19} \mathrm{O}_{3}[\mathrm{M}+\mathrm{H}]^{+}$235.1329, found 235.1336.

( $1 R, 2 S, 5 R)$-2-异丙基- 5 -甲基环己基 4-甲氧基苯甲酸 酯(3I) ${ }^{[23]}$ : 无色液体, 产率 $70 \% .{ }^{1} \mathrm{H}$ NMR $(400 \mathrm{MHz}$, DMSO- $\left.d_{6}\right) \delta: 7.92 \sim 7.88(\mathrm{~m}, 2 \mathrm{H}), 7.06 \sim 7.02(\mathrm{~m}, 2 \mathrm{H})$, $4.83 \sim 4.75(\mathrm{~m}, 1 \mathrm{H}), 3.82(\mathrm{~s}, 1 \mathrm{H}), 1.99 \sim 1.94(\mathrm{~m}, 1 \mathrm{H})$, $1.89 \sim 1.82(\mathrm{~m}, 1 \mathrm{H}), 1.70 \sim 1.64(\mathrm{~m}, 2 \mathrm{H}), 1.55 \sim 1.47(\mathrm{~m}$, $2 \mathrm{H}), 1.13 \sim 1.04(\mathrm{~m}, 2 \mathrm{H}), 0.91 \sim 0.86(\mathrm{~m}, 7 \mathrm{H}) ;{ }^{13} \mathrm{C} \mathrm{NMR}$ $\left(100 \mathrm{MHz}, \mathrm{DMSO}-d_{6}\right) \delta: 164.9,163.1,131.1,122.2,114.0$, 73.6, 46.6, 40.6, 33.7, 30.9, 26.2, 23.3, 21.9, 20.4, 16.5; HRMS (ESI) calcd for $\mathrm{C}_{18} \mathrm{H}_{26} \mathrm{O}_{3} \mathrm{Na}[\mathrm{M}+\mathrm{Na}]^{+} 313.1774$, found 313.1782 .

金刚烷-1-基甲基 4-甲氧基苯甲酸酯(3m) ${ }^{[18]}$ : 白色 固体, 产率 67\%. m.p. 106.5 107.9 ${ }^{\circ} \mathrm{C} .{ }^{1} \mathrm{H}$ NMR (400 MHz, DMSO- $\left.d_{6}\right) \delta: 7.95 \sim 7.91(\mathrm{~m}, 2 \mathrm{H}), 7.08 \sim 7.04(\mathrm{~m}$, $2 \mathrm{H}), 3.84(\mathrm{~s}, 2 \mathrm{H}), 3.83(\mathrm{~s}, 3 \mathrm{H}), 1.99 \sim 1.95(\mathrm{~m}, 3 \mathrm{H}), 1.72 \sim$ $1.62(\mathrm{~m}, 6 \mathrm{H}), 1.60 \sim 1.54(\mathrm{~m}, 6 \mathrm{H}) ;{ }^{13} \mathrm{C}$ NMR $(100 \mathrm{MHz}$, DMSO- $\left.d_{6}\right) \delta: 165.4,163.1,131.2,122.1,114.1,73.3,55.5$, 38.8, 36.4, 33.1, 27.4; HRMS (ESI) calcd for $\mathrm{C}_{19} \mathrm{H}_{24} \mathrm{O}_{3} \mathrm{Na}$ $[\mathrm{M}+\mathrm{Na}]^{+}$323.1618, found 323.1631.

(1S,2S,4S)-1,7,7-三甲基双环[2.2.1]庚烷-2-基 4-甲氧 基苯甲酸酯(3n) ${ }^{[24]}$ : 无色液体, 产率 $42 \% .{ }^{1} \mathrm{H}$ NMR (600 MHz, DMSO- $\left.d_{6}\right) \delta: 7.91 \sim 7.87(\mathrm{~m}, 2 \mathrm{H}), 7.05 \sim 7.01(\mathrm{~m}$, 2H), 4.72 (d, $J=4.72 \mathrm{~Hz}, 2 \mathrm{H}), 3.82$ (s, 3H), 2.34 (d, $J=$ $4.74 \mathrm{~Hz}, 1 \mathrm{H}), 2.30$ (t, $J=4.57 \mathrm{~Hz}, 1 \mathrm{H}), 1.77 \sim 1.74(\mathrm{~m}$, $1 \mathrm{H}), 1.67 \sim 1.34(\mathrm{~m}, 6 \mathrm{H}), 1.34 \sim 1.08(\mathrm{~m}, 7 \mathrm{H}), 0.93 \sim 0.83$ $(\mathrm{m}, 1 \mathrm{H}) ;{ }^{13} \mathrm{C}$ NMR (150 MHz, DMSO- $\left.d_{6}\right) \delta: 165.0,163.1$, 131.2, 122.4, 114.0, 77.1, 55.5, 41.1, 40.0, 39.2, 35.1, 34.9, 27.7, 23.8; HRMS (ESI) calcd for $\mathrm{C}_{18} \mathrm{H}_{25} \mathrm{O}_{3}[\mathrm{M}+\mathrm{H}]^{+}$ 289.1804 , found 289.1802 .

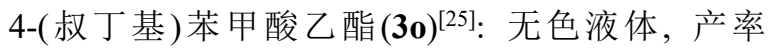
$85 \% .{ }^{1} \mathrm{H}$ NMR (600 MHz, $\left.\mathrm{CDCl}_{3}\right) \delta: 8.00 \sim 7.94(\mathrm{~m}, 2 \mathrm{H})$, $7.48 \sim 7.42(\mathrm{~m}, 2 \mathrm{H}), 4.36$ (q, $J=7.20 \mathrm{~Hz}, 2 \mathrm{H}), 1.38(\mathrm{t}, J=$ $7.07 \mathrm{~Hz}, 3 \mathrm{H}), 1.34$ (s, 9H); ${ }^{13} \mathrm{C}$ NMR $\left(150 \mathrm{MHz}, \mathrm{CDCl}_{3}\right) \delta$ : 166.7, 156.4, 129.4, 127.7, 125.3, 60.7, 35.0, 31.1, 14.3; HRMS (ESI) calcd for $\mathrm{C}_{13} \mathrm{H}_{19} \mathrm{O}_{2}[\mathrm{M}+\mathrm{H}]^{+}$207.1380, found 207.1377.

4-氯苯甲酸乙酯 $(3 \mathbf{p}){ }^{[26]}$ : 无色液体, 产率 $53 \% .{ }^{1} \mathrm{H}$ NMR $\left(600 \mathrm{MHz}\right.$, DMSO- $\left.d_{6}\right) \delta: 7.96 \sim 7.91(\mathrm{~m}, 2 \mathrm{H})$, $7.60 \sim 7.54(\mathrm{~m}, 2 \mathrm{H}), 4.30$ (t, $J=7.08 \mathrm{~Hz}, 2 \mathrm{H}), 1.31$ (t, $J=$
$7.13 \mathrm{~Hz}, 3 \mathrm{H}) ;{ }^{13} \mathrm{C}$ NMR (150 MHz, DMSO- $\left.d_{6}\right) \delta: 164.9$, 138.2, 131.0, 129.0, 61.1, 14.1; HRMS (ESI) calcd for $\mathrm{C}_{9} \mathrm{H}_{10} \mathrm{ClO}_{2}[\mathrm{M}+\mathrm{H}]^{+}$186.0448, found 186.0440 .

4-溴苯甲酸乙酯 $(3 q){ }^{[27]}$ : 无色液体, 产率 $67 \% .{ }^{1} \mathrm{H}$ NMR $\left(600 \mathrm{MHz}\right.$, DMSO- $\left.d_{6}\right) \delta: 7.89 \sim 7.86(\mathrm{~m}, 2 \mathrm{H}), 7$. $76 \sim 7.72(\mathrm{~m}, 2 \mathrm{H}), 4.31$ (t, $J=7.08 \mathrm{~Hz}, 2 \mathrm{H}), 3.35$ (s, $J=$ $7.07 \mathrm{~Hz}, 3 \mathrm{H}) .{ }^{13} \mathrm{C}$ NMR $\left(150 \mathrm{MHz}, \mathrm{DMSO}-d_{6}\right) \delta: 165.1$, 131.9, 131.1, 129.1, 127.3, 61.1, 14.1. HRMS (ESI) calcd for $\mathrm{C}_{9} \mathrm{H}_{10} \mathrm{BrO}_{2}[\mathrm{M}+\mathrm{H}]^{+}$228.9858, found 228.9849 .

4-甲酰基苯甲酸乙酯(3r $)^{[23]}$ : 无色液体，产率 $50 \%$. ${ }^{1} \mathrm{H}$ NMR (400 MHz, DMSO- $d_{6}$ ) $\delta: 10.11(\mathrm{~s}, 1 \mathrm{H}), 8.17 \sim$ $8.13(\mathrm{~m}, 2 \mathrm{H}), 8.06 \sim 8.03(\mathrm{~m}, 2 \mathrm{H}), 4.36(\mathrm{q}, J=7.07 \mathrm{~Hz}$, $2 \mathrm{H}), 1.34(\mathrm{t}, J=7.16 \mathrm{~Hz}, 3 \mathrm{H}) ;{ }^{13} \mathrm{C}$ NMR $(100 \mathrm{MHz}$, DMSO- $\left.d_{6}\right) \delta: 193.0,165.0,139.1,134.6,129.8,129.7$, 61.4, 14.1; HRMS (ESI) calcd for $\mathrm{C}_{10} \mathrm{H}_{11} \mathrm{O}_{3}[\mathrm{M}+\mathrm{H}]^{+}$ 179.0703, found 179.0709.

4-乙炔基苯甲酸乙酯(3s) ${ }^{[28]}$ : 无色液体, 产率 $58 \%$. ${ }^{1} \mathrm{H}$ NMR (400 MHz, DMSO- $\left.d_{6}\right) \delta: 7.97 \sim 7.93(\mathrm{~m}, 2 \mathrm{H})$, $7.64 \sim 7.60(\mathrm{~m}, 2 \mathrm{H}), 4.49(\mathrm{~s}, 1 \mathrm{H}), 4.31(\mathrm{q}, J=7.11 \mathrm{~Hz}$, $2 \mathrm{H}), 3.82$ (t, $J=7.11 \mathrm{~Hz}, 3 \mathrm{H}) ;{ }^{13} \mathrm{C}$ NMR $(100 \mathrm{MHz}$, DMSO- $\left.d_{6}\right) \delta: 165.1,132.0,129.9,129.3,126.4,84.0,82.6$, 61.0, 14.1; HRMS (ESI) calcd for $\mathrm{C}_{11} \mathrm{H}_{10} \mathrm{O}_{2} \mathrm{Na}[\mathrm{M}+\mathrm{Na}]^{+}$ 197.0573, found 197.0578.

辅助材料(Supporting Information) 化合物 $3 \mathrm{a} \sim 3 \mathrm{~s}$ 的 ${ }^{1} \mathrm{H} N M R$ 和 ${ }^{13} \mathrm{C}$ NMR 谱图. 这些材料可以免费从本刊网 站(http://sioc-journal.cn/)上下载.

\section{References}

[1] Hettikankanamalage, A. A.; Lassfolk, R.; Ekholm, F. S.; Leino, R.; Crich, D. Chem. Rev. 2020, 120, 7104.

[2] Larock, R. C. Comprehensive Organic Transformations: A Guide to Functional Group Preparations, John Wiley \& Sons, Inc., New York, 1999.

[3] Otera, J.; Nishikido, J. J. Am. Chem. Soc. 2010, 132, 9221.

[4] Sable, V.; Shah, J.; Sharma, A.; Kapdi, A. R. Chem. Asian J. 2019, 14, 2639.

[5] Endo, A.; Kuroda, M.; Tsujita, Y. J. Antibiot. 1976, 29, 1346.

[6] (a) Liu, C.; Tang, S.; Zheng, L.; Liu, D.; Zhang, H.; Lei, A. Angew. Chem., Int. Ed. 2012, 51, 5662.

(b) Zhu, L.; Ren, X.; Yu, Y.; Ou, P.; Wang, Z.-X.; Huang, X. Org. Lett. 2020, 22, 2087.

(c) Li, X.; Goh, T. W.; Li, L.; Xiao, C.; Guo, Z.; Zeng, X. C.; Huang, W. ACS Catal. 2016, 6, 3461.

(d) Suzuki, K.; Yamaguchi, T.; Matsushita, K.; Iitsuka, C.; Miura, J.; Akaogi, T.; Ishida, H. ACS Catal. 2013, 3,1845.

(e) Paul, B.; Khatun, R.; Sharma, S. K.; Adak, S.; Singh, G.; Das, D.; Siddiqui, N.; Bhandari, S.; Joshi, V.; Sasaki, T.; Bal, R. ACS Sustainable Chem. Eng. 2019, 7, 3982.

(f) Cheng, J.; Zhu, M.; Wang, C.; Li, J.; Jiang, X.; Wei, Y.; Tang, W.; Xue, D.; Xiao, J. Chem. Sci. 2016, 7, 4428.

[7] (a) Sarkar, S. D.; Grimme, S.; Studer, A. J. Am. Chem. Soc. 2010, 132, 1190.

(b) Liu, B.; Yan, J.; Huang, R.; Wang, W.; Jin, Z.; Zanoni, G.; 
Zheng, P.; Yang, S.; Chi, Y. R. Org. Lett. 2018, 20, 3447.

(c) Wu, Z.; Jiang, D.; Wang, J. Org. Chem. Front. 2019, 6, 688.

(d) Carmine, G. D.; Ragno, D.; Massi, A.; D'Agostino, C. Org. Lett. 2020, 22, 4927.

[8] (a) Gaspa, S.; Porcheddu, A.; Luca, L. D. Org. Lett. 2015, 17, 3666.

(b) Chun, S.; Chung, Y. K. Org. Lett. 2017, 19: 3787

(c) Mühldorf, B.; Wolf, R. ChemCatChem 2017, 9, 920 .

(d) Kozlov, K. S.; Romashov, L. V.; Ananikov, V. P. Green Chem. 2019, 21, 3464

[9] (a) Yan, M.; Kawamata, Y.; Baran, P. S. Chem. Rev. 2017, 117, 13230.

(b) Moeller, K. D. Chem. Rev. 2018, 118, 4817

(c) Wiebe, A.; Gieshoff, T.; Möhle, S.; Rodrigo, E.; Zirbes, M.; Waldvogel, S. R. Angew. Chem., Int. Ed. 2018, 57, 5594.

(d) Francke, R.; Little, R. D. Chem. Soc. Rev. 2014, 43, 2492.

(e) Yoshida, J.; Shimizu, A.; Hayashi, R. Chem. Rev. 2018, 118, 4702

(f) Tang, S.; Liu, Y.; Lei, A. Chem 2018, 4, 27.

(g) Yuan, Y.; Lei, A. Acc. Chem. Res. 2019, 52, 3309

(h) Xiong, P.; Xu, H.-C. Acc. Chem. Res. 2019, 52, 3339.

(i) Jiang, Y.; Xu, K.; Zeng, C. Chem. Rev. 2018, 118, 4485.

(j) Zhou, Y.-J.; Zhao, Z.-H.; Zeng, L.; Li, L.; He, Y.-H.; Gu, L.-J. Chin. J. Org. Chem. 2021, 41, 1072 (in Chinese).

(周娅琴, 赵志恒, 曾亮, 李鸣, 何永辉, 谷利军, 有机化学, 2021, 41, 1072.)

(k) Meng, Z.-Y.; Feng, C.-T.; Xu, K. Chin. J. Org. Chem. 2021, 41, 2535 (in Chinese)

(蒙泽银, 冯承涛, 徐坤, 有机化学, 2021, 41, 2535.)

(1) Wu, M.; Yu, L.; Hou, H.-Q.; Chen, H.-Z.; Zhuang, Q.-L.; Zhou, S.-Y.; Lin, X.-Y. Chin. J. Org. Chem. 2021, 41, 2326 (in Chinese). (吴媚, 于玲, 侯慧青, 陈厚铮, 庄庆龙, 周孙英, 林小燕, 有机 化学, 2021, 41, 2326.)

(m) (m)Liu, W.-Q.; Yang, X.-L.; Tong, Z.-H.; Wu, L.-Z. Acta Chim. Sinica 2019, 77, 861 (in Chinese).

(刘文强, 杨修龙, 佟振合, 吴骊珠, 化学学报, 2019, 77, 861.)

(n) Ma, Y.; Wu, S.; Jiang, S.; Xiao, F.; Deng, G.-J. Chin. J. Chem. 2021, 39, 3334

(o) Yang, Z.; Yu, Y.; Lai, L.; Zhou, L.; Ye, K.; Chen, F.-E. Green Synth. Catal. 2021, 2, 19.

(p) Chen, N.; Xu, H.-C. Green Synth. Catal. 2021, 2, 165.

(q) Wu, Y.; Chen, J.-Y.; Liao, H.-R.; Shu, X.-R.; Duan, L.-L.; Yang,

X.-F.; He, W.-M. Green Synth. Catal. 2021, 2, 233.

(r) Chen, J.-Y.; Wu, H.-Y.; Gui, Q.-W.; Yan, S.-S.; Deng, J.; Lin, Y.-W.; Cao, Z.; He, W.-M. Chin. J. Catal. 2021, 42, 1445.

[10] Finney, E. E.; Ogawa, K. A.; Boydston, A. J. J. Am. Chem. Soc.
2012, 134, 12374 .

[11] Green, R. A.; Pletcher, D.; Leach, S. G.; Brown, R. C. D. Org. Lett. 2015, 17, 3290 .

[12] (a) Zhong, P.-F.; Lin, H.-M.; Wang, L.-W.; Mo, Z.-Y.; Meng, X.-J.; Tang, H.-T.; Pan, Y.-M. Green Chem. 2020, 22, 6334.

(b) He, M.-X.; Mo, Z.-Y.; Wang, Z.-Q.; Cheng, S.-Y.; Xie, R.-R.; Tang, H.-T.; Pan, Y.-M. Org. Lett. 2020, 22, 724.

(c) Li, Q.-Y.; Cheng, S.-Y.; Tang, H.-T.; Pan, Y.-M. Green Chem. 2019, 21, 5517.

(d) Meng, X.-J.; Zhong, P.-F.; Wang, Y.-M.; Wang, H.-S.; Tang, H.-T.; Pan, Y.-M. Adv. Synth. Catal. 2020, 362, 506.

(e) Zhang, Y.-Z.; Mo, Z.-Y.; Wang, H.-S.; Wen, X.-A.; Tang, H.-T.; Pan, Y.-M. Green Chem. 2019, 21, 3807.

(f) Mo, Z.-Y.; Swaroop, T. R; Tong, W.; Zhang, Y.-Z.; Tang, H.-T.; Pan, Y.-M.; Sun, H.-B.; Chen, Z.-F. Green Chem. 2018, 20, 4428.

[13] Sawamura, T.; Takahashi, K.; Inagi, S.; Fuchigami, T. Angew. Chem., Int. Ed. 2012, 51, 4413.

[14] Xie, C.; Lin, L., Huang, L.; Wang, Z.; Jiang, Z.; Zhang, Z.; Han, B. Nat. Commun. 2021, 12, 4823.

[15] Teng, B.; Shi, J.; Yao, C. Green Chem. 2018, 20, 2465.

[16] Yu, D.; To, W.-P.; Tong, G. S. M.; Wu, L.-L.; Chan, K.-T.; Du,L.; Phillips, D. L.; Liu, Y.; Che, C.-M. Chem. Sci. 2020, 11, 6370.

[17] Wang, L.; Neumann, H.; Spannenberg, A.; Beller, M. Chem. Commun. 2017, 53, 7469 .

[18] Yang, H. S.; Macha, L.; Ha, H.-J.; Yang, J. W. Org. Chem. Front. 2021, 8,53 .

[19] Khosravi, K.; Khalaji, K.; Naserifar, S. J. Chin. Chem. Soc. 2017, 64,303

[20] Yu, C.; Özkaya, B.; Patureau. F. W. Chem.-Eur. J. 2021, 27, 3682.

[21] Lawson, J. R.; Wilkins, L. C.; Melen, R. L. Chem.-Eur. J. 2017, 23, 10997.

[22] Veatch, A. M.; Alexanian, E. J. Chem. Sci. 2020, 11, 7210

[23] Bourne-Branchu, Y.; Gosmini, C.; Danoun, G. Chem.-Eur. J. 2017, $23,10043$.

[24] Silva, A. T. M.; Pereira, V. V.; Takahashi, J. A.; Silva, R. R.; Duarte, L. P. Nat. Prod. Res. 2018, 32, 1714 .

[25] Hoque, M. E.; Hassan, M. M. M.; Chattopadhyay, B. J. Am. Chem. Soc. 2021, 143, 5022.

[26] Rysak, V.; Dixit, R.; Trivelli, X.; Merle, N.; Agbossou-Niedercorn, F.; Kumar, V.; Michon, C. Cat. Sci. Technol. 2020, 10, 4586.

[27] Rammurthy, B.; Peraka, S.; Vasu, A.; Sai, G. K.; Rohini, Y. D.; Narender, N. Asian J. Org. Chem. 2021, 10, 594.

[28] Velasco, N.; Suárez, A.; Martínez-Lara, F.; Fernández-Rodríguez, M. Á.; Sanz, R.; Suárez-Pantiga, S. J. Org. Chem. 2021, 86, 7078. 